\title{
Estructura genética poblacional del pez Brycon henni (Characiformes: Characidae) en la región andina de Colombia utilizando marcadores microsatélites
}

\author{
Luz Elena Muñoz-A. ${ }^{1}$, Julia Victoria Arredondo-B. ${ }^{2}$ \& Jaime Andrés Carranza-Quiceno ${ }^{1 *}$ \\ 1. Facultad de Ciencias Básicas, Corporación universitaria Santa Rosa de Cabal, UNISARC, Km 4 Vía Santa Rosa de \\ Cabal - Chinchiná. Santa Rosa de Cabal - Risaralda - Colombia; biologia@unisarc.edu.co, \\ jaime.carranza@unisarc.edu.co \\ 2. Facultad de Ciencias Pecuarias, Corporación universitaria Santa Rosa de Cabal, UNISARC, Km 4 Vía Santa Rosa de \\ Cabal - Chinchiná. Santa Rosa de Cabal - Risaralda - Colombia; victoria.arredondo@unisarc.edu.co \\ * Correspondencia
}

Recibido 26-II-2018. Corregido 14-V-2019. Aceptado 20-VI-2019.

\begin{abstract}
Population genetic structure of Brycon henni fish (Characiformes: Characidae) in the Andean region of Colombia using microsatellite molecular markers. Fish species in Colombian Andes are subject to processes of overfishing, exotic species invasions, habitat loss, ecosystems fragmentation and low connectivity that limits the genetic flow and leads populations to loss genetic variability and extinction. Genetic variation of B. henni from four basins of the Colombian Andean region (Risaralda, Campoalegre, Riofrío and Chinchiná) was analyzed using ten microsatellites in 60 samples. A total of 136 alleles were found. Except BoM12, all markers were highly informative and polymorphic and presented a significant deviation $(\mathrm{P}<0.05)$ from Hardy Weinberg Equilibrium. There was a marked deficiency of heterozygotes $\left(\mathrm{F}_{\mathrm{IS}}=0.526\right)$. In Campoalegre river basin, the largest allele number, average allele number and private alleles were observed (81, eight and 34 alleles, respectively) and $\mathrm{H}_{\mathrm{E}} 0.689 \pm 0.05$. Moderate and highly significant genetic structure was evidenced $\left(\mathrm{F}_{\mathrm{ST}}\right.$ $=0.07$ ). According to the standard distance of Nei (1972), population from Campoalegre River basin was the most divergent. This results suggest that genetic variability of the $B$. henni in the studied basins may be affected by critical low population density, river pollution and overfishing.
\end{abstract}

Key words: Andean region; codominant marker; genetic variability; overfishing.

Muñoz-A., L. E., Arredondo-B., J. V., \& Carranza-Quiceno, J. A. (2019). Estructura genética poblacional del pez Brycon henni (Characiformes: Characidae) en la región andina de Colombia utilizando marcadores microsatélites. Revista de Biología Tropical, 67(4), 733-744.

El género Brycon (Characiformes: Characidae), consta de 42 especies y se distribuye desde el sur de México al Río La Plata en Argentina, con mayor diversidad en los ríos de Panamá y los ríos transandinos de Colombia y Ecuador (Silva, Oliveira, De Lima, \& Matsumoto, 2008); este género comprende la especie endémica de Colombia Brycon henni
(Eigenmann, 1913), conocida como "Sabaleta", presente en los principales afluentes de las cuencas de los ríos Cauca, Magdalena, San Juan, Dagua, Patía y San Jorge (Montoya, Carrillo, \& Olivera, 2006a) y acuíferos de áreas productoras de café de la región central de Colombia. B. henni tiene una gran significación cultural en las zonas donde ocurre (Lenis, Cruz, 
\& David, 2015), principalmente por la calidad de su carne y su comportamiento en la pesca deportiva (Pineda et al., 2007).

La especie ha sido estudiada desde el punto de vista biológico, anatómico y ecológico (Maldonado et al., 2005; Montoya et al., 2006b; Botero \& Ramírez, 2011), productivo y reproductivo (Tabares, Ruíz, Arboleda, \& Olivera, 2006; Tabares, Ruíz, Arboleda, \& Olivera, 2007; Lenis, Restrepo, \& Cruz, 2009; Chacón, Angel, Serna, Echavarría, \& Vélez, 2015; Aguirre \& Muñoz, 2015; Lenis et al., 2015), citogenético (David, Vásquez, Ruiz, \& Olivera, 2008; Muñoz, Villamarín, \& Londoño, 2016; De Miranda et al., 2014) y molecular, con marcadores moleculares RAPD (Pineda et al., 2007; Hurtado, Mancera, \& Saldamando, 2011). A pesar de ello, el conocimiento sobre algunos aspectos aun es fragmentado y no hay estudios de variabilidad genética basados en marcadores moleculares codominantes, como los microsatélites, los cuales han sido ampliamente usados en otras especies de Brycon, como Brycon opalinus (Cuvier, 1819) por Barroso, Hilsdorf, Moreira, Cabellod, y Traub (2005), Brycon orbignyanus (Eigenmann \& Norris, 1900) por Rodriguez et al. (2010); Lopera et al. (2010); Moreira, Polo, Silva, y Menezes (2015), Brycon insignis (Eigenmann \& Norris, 1900) por Matsumoto y Silva (2009), Brycon hilarii (Valenciennes, 1850) por Sanches \& Galetti (2012) y Brycon orthotaenia (Günther, 1864) por Sanches et al. (2012).

Los niveles de variabilidad genética de las poblaciones naturales de peces pueden ser afectados por características del ambiente en el que viven o bien pueden reflejar los eventos ambientales históricos que han sucedido en la región que ocupan (Palma, González, Romo, Ruiz, \& Fuentealba, 2007). Según Mancera et al. (2013) las especies de peces en Colombia están sujetas a procesos de sobrepesca, introducción de especies exóticas, deterioro de hábitat y fragmentación de los ecosistemas, la cual limita el flujo genético entre las poblaciones. El monitoreo de la diversidad genética de las poblaciones naturales de peces es importante para su conservación; los marcadores moleculares son herramientas útiles para ello (Povh et al., 2008) y los microsatélites son los más ampliamente utilizados, especialmente en estudios genéticos de poblaciones de peces tropicales de agua dulce (Piorski et al., 2008), ya que permiten comparar entre poblaciones, dadas sus propiedades de abundancia, hipervariabilidad y herencia mendeliana (Ellegren, 2004; Kumar, Tomar, Kumar, \& Singh, 2017).

En la cuenca alta del río Cauca, no se han realizado estudios sobre el estado de las poblaciones de $B$. henni que habitan en sus afluentes, no obstante, esta es una de las regiones de Colombia con mayor desarrollo de la agroindustria y la actividad minera; muchos de estos cauces son objeto de extracción de piedra $\mathrm{y}$ arena, y se han establecido represas para la generación hidroeléctrica y las actividades recreativas (CVC, 2004). Aunque $B$. henni no se encuentra en ninguna de las categorías de riesgo propuestas por el libro rojo de peces dulceacuícolas de Colombia (Mojica, Usma, Álvarez-León, \& Lasso, 2012), la alteración de su hábitat y el aislamiento de las poblaciones por el deterioro de la calidad del agua del río Cauca pueden tener un efecto sobre la estructura genética de las poblaciones, con consecuencias sobre su conservación (Hurtado et al., 2011). Este estudio tuvo como objetivo analizar la estructura genética poblacional de $B$. henni en cuatro cuencas de la región Andina colombiana, utilizando marcadores moleculares microsatélites e identificar las poblaciones con prioridad de conservación.

\section{MATERIALES Y MÉTODOS}

Recolección de muestras: Se obtuvieron 60 individuos de $B$. henni, distribuidos en cuatro cuencas hidrográficas de la región Andina de Colombia (quince en cada una). Estas fueron los ríos Risaralda (RR) $\left(05^{\circ} 06^{\prime} \mathrm{N}-75^{\circ} 50^{\prime}\right.$ $\mathrm{W})$, Campoalegre (RC) $\left(4^{\circ} 56^{\prime} \mathrm{N}-75^{\circ} 37^{\prime} \mathrm{W}\right)$, Riofrío (RRf) $\left(04^{\circ} 07^{\prime} \mathrm{N}-76^{\circ} 21^{\prime} \mathrm{W}\right)$ y Río Chinchiná (RCh) $\left(5^{\circ} 03^{\prime} \mathrm{N}-75^{\circ} 36^{\prime} \mathrm{W}\right)$. Los individuos fueron capturados con métodos de pesca artesanal (atarraya) y la especie fue determinada de acuerdo a Maldonado et al. (2005). 
Procedimientos de laboratorio: $100 \mathrm{mg}$ de músculo esquelético de los animales muestreados se almacenaron en etanol al $95 \%$ hasta su uso. El ADN genómico se aisló usando el protocolo de extracción con Fenol-Cloroformo, de acuerdo con Taggart, Hynes, Prodohl \& Fergusson (1992) modificando el tipo de enzima proteolítica y el detergente iónico, para ello se resuspendieron $100 \mathrm{mg}$ de musculo esquelético en $375 \mu \mathrm{l}$ de EDTA (ácido etilendiamino tetraacético) $0.2 \mathrm{M}, 1 \%$ de SDS y $7 \mu \mathrm{L}$ de proteinasa $\mathrm{K}(200 \mu \mathrm{g} / \mathrm{ml})$, seguida por incubación a $37^{\circ} \mathrm{C}$ por $15 \mathrm{~h}$. Posteriormente se adicionaron $10 \mu \mathrm{l}$ de DNAsa $(2 \mathrm{mg} / \mathrm{ml})$ y se incubaron a $37^{\circ} \mathrm{C}$ por $1 \mathrm{~h}$. Se adicionaron $400 \mu \mathrm{l}$ de fenol ( $\mathrm{pH} 8.0)$, con agitación vigorosa por $20 \mathrm{~s}$ y luego más suavemente por $10 \mathrm{~min}$; se adicionaron $400 \mu \mathrm{l}$ de alcohol cloroformo: isoamilico (24:1) a cada tubo, y se realizó la misma agitación seguida de centrifugación a $12000 \mathrm{~g}$ por $3 \mathrm{~min}$. Luego el sobrenadante fue mezclado por inversión de 5 a 6 veces con etanol frio al $99 \%$ por $15 \mathrm{~min}$, seguido por un lavado con etanol al $70 \%$ por $1 \mathrm{~h}$ con mezclado suave. Finalmente se decantó el etanol y se dejó secar el ADN por $10 \mathrm{~min}$ a temperatura ambiente y se resuspendió en buffer TE (10 mM Tris, 1 mM EDTA; pH 8.0).

El ADN fue cuantificado en un nanodrop 2000 Thermo Sientific y su integridad verificada con electroforesis horizontal en un gel de agarosa a una concentración del $1 \%$. Se utilizaron doce cebadores (Tabla 1) previamente evaluados en otras especies de Brycon: Bh17, Bh5, Bh6, Bh8, Bh15 (Sanches \& Galetti, 2006), BoM1, BoM2, BoM5, BoM6, BoM7, BoM12 y BoM13 (Barroso et al., 2003). Su síntesis, amplificación y secuenciación fue realizada mediante un secuenciador ABI3730XL

TABLA 1

Información de los microsatélites empleados en este estudio. Brycon opalinus (Bom1, Bom2, Bom5, Bom6, Bom7, Bom12, Bom13) y Brycon hilarii (Bh5, Bh6, Bh8, Bh15, Bh17)

TABLE 1

Microsatellites information used in this study. Brycon opalinus (Bom1, Bom2, Bom5, Bom6, Bom7, Bom12, Bom13) and Brycon hilarii (Bh5, Bh6, Bh8, Bh15, Bh17)

\begin{tabular}{|c|c|c|c|c|}
\hline \multirow{2}{*}{ Locus } & \multirow{2}{*}{ Secuencia del cebador $\left(5^{\prime}-3^{\prime}\right)$} & \multirow{2}{*}{ Motivo- repetición } & \multicolumn{2}{|c|}{ Rango alélico (pb) } \\
\hline & & & Reportado (pb) & Encontrado $(\mathrm{pb})$ \\
\hline Bh17 & $\begin{array}{l}\text { F: GTCAGCACTCAGCACATAGC } \\
\text { R: AGAGAGCCTGAAAGTGAGTC }\end{array}$ & $(\mathrm{GTTT})_{4}(\mathrm{GGTTT})_{3}$ & $152-212$ & $150-225$ \\
\hline Bh5 & $\begin{array}{l}\text { F: CTTCCACTCATACCGGCACT } \\
\text { R: ACATCTGGCATTAGGCATAG }\end{array}$ & $(\mathrm{AC})_{13}$ & $204-220$ & $164-226$ \\
\hline Bh6 & $\begin{array}{l}\text { F: GCGTTGCGTGTGTATGTTAA } \\
\text { R: AGAGGTGTCCACAAAGTTTT }\end{array}$ & $(\mathrm{GT})_{14}$ & $160-184$ & $168-193$ \\
\hline Bom1 & $\begin{array}{l}\text { F: CCATCTCTACTTTTTGGTTCC } \\
\text { R: TGCCCAGATACAGCCC }\end{array}$ & $(\mathrm{CA})_{16}$ & $139-173$ & $127-212$ \\
\hline Bom 12 & $\begin{array}{l}\text { F: GCAGCAGAAAGAAACAG } \\
\text { R: CGGGGAGATTTCAACCT }\end{array}$ & $(\mathrm{GT})_{15}$ & $78-118$ & $55-118$ \\
\hline Bom 13 & $\begin{array}{l}\text { F: CATTTCCTCAGTCCTTTTCAGC } \\
\text { R: CCCACTTAGGGTCGCAC }\end{array}$ & $(\mathrm{CT})_{11}$ & $154-176$ & $158-192$ \\
\hline Bom2 & $\begin{array}{l}\text { F: CTGGGCAGCGGAAGAG } \\
\text { R: CCCACATCTCTCCCCTTCG }\end{array}$ & $(\mathrm{CA})_{45}$ & $162-242$ & $216-240$ \\
\hline Bom5 & $\begin{array}{l}\text { F: CGACCACAATAGGATTAGGG } \\
\text { R: CTGGAGTTTGTGTGTGGA }\end{array}$ & $(\mathrm{AC})_{4} \mathrm{~T}(\mathrm{AC})_{10} \mathrm{AT}(\mathrm{AC})_{5}$ & $117-151$ & $129-165$ \\
\hline Bom6 & $\begin{array}{l}\text { F: GGAGTTTGTGTGTGGAGACCGAG } \\
\text { R: GCACGCAGACACCAGA }\end{array}$ & $\mathrm{TA}(\mathrm{CA})_{10} \mathrm{~T}(\mathrm{CA})_{4}$ & $142-178$ & $125-188$ \\
\hline Bom7 & $\begin{array}{l}\text { F: CTCTGCCCCAGGTCTCACT } \\
\text { R: CGGGAGTGACGAAATG }\end{array}$ & $(\mathrm{CA})_{31}$ & $172-236$ & $164-225$ \\
\hline
\end{tabular}

Barroso et al. (2003), Sanches \& Galetti (2006). 
(Macrogen Sequencing Service, Corea) y el tamaño de los alelos se midió mediante el software Peak scanner TM V.1.0.

Informatividad de los microsatélites y características de las poblaciones: Los marcadores empleados en el presente estudio fueron seleccionados por haber sido los más polimórficos en investigaciones previas con otras especies de Brycon (Sanches \& Galetti, 2006; Matsumoto \& Silva, 2009). Rodríguez et al., (2010), Sanches y Galetti (2012), Lopera et al., (2010) y Sanches et al. (2012), que evaluaron 5, 7, 4 y 5 loci en las especies $B$. opalinus, $B$. hilarii, B. orbignyanus y B. orthotaenia, respectivamente. Los microsatélites BoM2 y BoM7 han mostrado alto polimorfismo en estudios realizados en B. opalinus, con 31 y 25 alelos respectivamente (Barroso et al., 2003).

Los marcadores fueron descritos mediante la estimación de parámetros como el número de alelos $\left(\mathrm{N}_{\mathrm{A}}\right)$, el número promedio y efectivo de alelos $\left(\mathrm{NP}_{\mathrm{A}}\right.$ y $\left.\mathrm{NE}_{\mathrm{A}}\right)$, la heterocigosidad observada $\left(\mathrm{H}_{\mathrm{O}}\right)$ y esperada $\left(\mathrm{H}_{\mathrm{E}}\right)$, el coeficiente de endogamia $\left(\mathrm{F}_{\mathrm{IS}}\right)$, las desviaciones del equilibrio Hardy Weinberg (EHW) dentro de las poblaciones y número privado de alelos para cada población $\left(\mathrm{NP}_{\mathrm{A}}\right)$ con el programa GenAlex (Peakall \& Smouse, 2006). Se estimó la riqueza alélica $\left(\mathrm{R}_{\mathrm{A}}\right)$ utilizando el programa FSTAT (Goudet, 2002) y el contenido de información polimórfica (PIC) con la aplicación Microsatellite Toolkit para Excel (Park, 2001).

Estructura genética: Fue analizada mediante los estadísticos de estructura poblacional de Wright $\left(\mathrm{F}_{\text {IT }}, \mathrm{F}_{\mathrm{ST}}\right.$ y $\left.\mathrm{F}_{\mathrm{IS}}\right)$ y el AMOVA basado en el estadístico $\mathrm{R}_{\mathrm{ST}}$ asumiendo un modelo de mutación por pasos (SMM), que refleja más exactamente el patrón de mutación de los microsatélites (Balloux \& Lugon-Moulin, 2002), así como la prueba de desequilibrio de ligamiento con 10000 permutaciones, mediante el programa Arlequin (Excoffier, Laval \& Scheneider, 2005). Las distancias genéticas entre las poblaciones se calcularon como Distancia de Nei (Da) (Nei, 1972) y se construyó un dendrograma basado en la
UPGMA (Unweighted Pair Group Method with Arithmetic mean) con TFPGA (Miller, 1997), para representar gráficamente esas distancias. Por último, se utilizó el programa Structure (Pritchard, Stephens, \& Donnelly, 2000) para asignar individuos a los grupos por similitud genética, mediante métodos bayesianos. Se tomaron diferentes números de poblaciones, asumiendo el modelo de mezcla y las frecuencias alélicas correlacionadas, con 100000 a 500000 iteraciones y dos repeticiones para cada K, cuyo valor más probable fue determinado por el método de Evanno, Regnaut, y Goudet (2005), con el Software Structure Harvester (Earl \& VonHoldt, 2012).

\section{RESULTADOS}

Informatividad de microsatélites: Dos de los 12 marcadores seleccionados, Bh8 y Bh15, no amplificaron exitosamente. Las características de los 10 loci microsatélites analizados se presentan en la tabla 2.

Todos los loci fueron polimórficos, el valor más alto de $\mathrm{N}_{\mathrm{A}}$ se presentó en BoM1, BoM2 y BoM7, con 20, 20 y 16 alelos totales, respectivamente. El $\mathrm{NP}_{\mathrm{A}}$ general fue de 6.45 , con valores por cada locus que van desde 4 a 8.75. Todos los marcadores fueron informativos y variables, excepto BoM12, con PIC de 0.39 , considerado razonablemente informativo (Botstein, White, Skolnick, \& Davis, 1980) y una $\mathrm{H}_{\mathrm{E}}$ inferior a 0.5 . La $\mathrm{R}_{\mathrm{A}}$, que estima la diversidad alélica de una población independientemente del número de muestras analizadas, osciló entre 3.75 y 6.99 .

Todos los marcadores mostraron déficit de heterocigotos. Se presentaron valores de $\mathrm{F}_{\mathrm{IS}}$ entre 0.242 y 0.722 ; el índice de fijación genética $\mathrm{F}_{\mathrm{IT}}$ mostró una fuerte desviación respecto a la situación de panmixia (0.504) y se evidenció una desviación significativa de EHW (P < 0.05), dada posiblemente por la probabilidad de alelos nulos, que en los loci analizados se encuentra entre 0.088 y 0.26 . De los diez loci analizados, cuatro presentaron alta probabilidad de ocurrencia de alelos nulos, con valores de $\mathrm{r}>0.2$ (Bh5, BoM1, BoM5, y BoM6), 
TABLA 2

Resumen estadístico para los loci microsatélites analizados

TABLE 2

Stats of microsatellites loci analyzed

\begin{tabular}{lcccccccccccc} 
Locus & $\mathrm{N}_{\mathrm{A}}$ & $\mathrm{NP}_{\mathrm{A}}$ & $\mathrm{NE}_{\mathrm{A}}$ & $\mathrm{R}_{\mathrm{A}}$ & $\mathrm{PIC}$ & $\mathrm{H}_{\mathrm{E}}$ & $\mathrm{H}_{\mathrm{O}}$ & $\mathrm{F}_{\mathrm{IS}}$ & $\mathrm{F}_{\mathrm{IT}}$ & $\mathrm{F}_{\mathrm{ST}}$ & $\mathrm{r}$ & $\mathrm{EHW}$ \\
Bh17 & 11 & 5.5 & 2.581 & 5.291 & 0.655 & 0.572 & 0.339 & 0.407 & 0.503 & 0.162 & 0.148 & 3 \\
Bh5 & 10 & 5.2 & 3.450 & 5.277 & 0.701 & 0.703 & 0.354 & 0.497 & 0.520 & 0.046 & 0.205 & 3 \\
Bh6 & 9 & 5.5 & 3.891 & 5.358 & 0.750 & 0.741 & 0.533 & 0.280 & 0.318 & 0.053 & 0.119 & 2 \\
BoM1 & 20 & 8.2 & 3.945 & 6.512 & 0.720 & 0.701 & 0.350 & 0.501 & 0.531 & 0.061 & 0.206 & 3 \\
BoM12 & 15 & 5.0 & 1.737 & 3.959 & 0.387 & 0.347 & 0.200 & 0.424 & 0.501 & 0.133 & 0.109 & 3 \\
BoM13 & 12 & 6.5 & 2.776 & 5.511 & 0.609 & 0.572 & 0.433 & 0.242 & 0.312 & 0.093 & 0.088 & 3 \\
BoM2 & 20 & 8.2 & 2.674 & 6.267 & 0.640 & 0.555 & 0.370 & 0.332 & 0.431 & 0.148 & 0.119 & 3 \\
BoM5 & 15 & 7.5 & 3.692 & 5.934 & 0.722 & 0.703 & 0.250 & 0.644 & 0.666 & 0.061 & 0.266 & 4 \\
BoM6 & 8 & 4.0 & 2.232 & 3.747 & 0.513 & 0.510 & 0.142 & 0.722 & 0.756 & 0.123 & 0.244 & 4 \\
BoM7 & 16 & 8.7 & 4.135 & 6.986 & 0.785 & 0.739 & 0.402 & 0.456 & 0.500 & 0.081 & 0.194 & 4 \\
\hline
\end{tabular}

Número de alelos $\left(\mathrm{N}_{\mathrm{A}}\right)$, Número promedio de alelos $\left(\mathrm{N}_{\mathrm{PA}}\right)$, Número efectivo de alelos $\left(\mathrm{NE}_{\mathrm{A}}\right)$, Riqueza alélica $\left(\mathrm{R}_{\mathrm{A}}\right)$, Contenido de información polimórfica $\left(\mathrm{P}_{\mathrm{IC}}\right)$, Heterocigosidad esperada $\left(\mathrm{H}_{\mathrm{E}}\right)$, Heterocigosidad observada $\left(\mathrm{H}_{\mathrm{O}}\right)$, Coeficiente de endogamia respecto a la subpoblación $\left(\mathrm{F}_{\mathrm{IS}}\right)$, coeficiente de endogamia total $\left(\mathrm{F}_{\mathrm{IT}}\right)$, Indice de diferenciación genética entre subpoblaciones $\left(\mathrm{F}_{\mathrm{ST}}\right)$, Frecuencia de alelos nulos $(\mathrm{r})$ y poblaciones desviadas del equilibrio Hardy-Weinberg (EHW) por locus.

Alleles number $\left(\mathrm{N}_{\mathrm{A}}\right)$, Average number of alleles $\left(\mathrm{N}_{\mathrm{PA}}\right)$, Effective number of alleles $\left(\mathrm{NE}_{\mathrm{A}}\right)$, Alelic Richness $\left(\mathrm{R}_{\mathrm{A}}\right)$, Polymorphic information contain $\left(\mathrm{P}_{\mathrm{IC}}\right)$, expected heterozygosity $\left(\mathrm{H}_{\mathrm{E}}\right)$, observed heterozygosity $\left(\mathrm{H}_{\mathrm{O}}\right)$, Coefficient of inbreeding with respect to the subpopulation $\left(\mathrm{F}_{\mathrm{IS}}\right)$, Total coefficient of inbreeding $\left(\mathrm{F}_{\mathrm{IT}}\right)$, genetic differentiation index between subpopulations $\left(\mathrm{F}_{\mathrm{ST}}\right)$, Null aleles frequency $(\mathrm{r})$ y populations deviated from Hardy-Weinberg equilibrium (EHW) by locus.

mientras que la probabilidad en los loci restantes fue moderada, con valores de $0.05<\mathrm{r}<0.2$.

Se estimaron las desviaciones del desequilibrio de ligamiento para evaluar la independencia de genotipos entre los loci, pues la presencia de endogamia en una población generaría correlación entre todos los loci (desequilibrio por ligamiento) (Gastric, Bernatchez, Belkhir, \& Bonhome, 2002). Se encontraron desequilibrios de ligamiento (DL) significati$\operatorname{vos}(\mathrm{P}<0.05)$ entre algunas parejas de marcadores; los marcadores que presentaron mayor DL en todas sus comparaciones fueron el Bh17 y el Bh5. La población que presentó mayor número de pares de marcadores en DL, fueron RRf y RCh, con 31 y 28 parejas de marcadores respectivamente, mientras que RR fue la población que menos marcadores en desequilibrio mostró, con 6 parejas.

Variación genética: Se encontraron un total de 136 alelos en todas las poblaciones.
La población de la cuenca del Río Campoalegre $(\mathrm{RC})$ presentó los mayores $\mathrm{N}_{\mathrm{A}}(81), \mathrm{NP}_{\mathrm{A}}$ (8), $\mathrm{A}_{\mathrm{P}}(34) \mathrm{y}_{\mathrm{E}}$ de $0.689 \pm 0.05$, mientras que la población de la cuenca del Río Riofrío (RRf) presentó los valores más bajos, con 53, 5 y 10 alelos respectivamente y $\mathrm{H}_{\mathrm{E}}$ de $0.515 \pm$ 0.055 (Tabla 3).

Se encontró un total de $78 \%$ de alelos con frecuencia menor a $10 \%$; se puede inferir la pérdida de variabilidad genética debido a la disminución de los alelos. Todas las poblaciones presentaron déficit de heterocigotos. La mayor diferencia entre ambas heterocigosidades se presentó en $\mathrm{RCh}$, con $\mathrm{F}_{\mathrm{IS}}$ de 0.551 y la menor en $\mathrm{RC}$, con $\mathrm{F}_{\mathrm{IS}}$ de 0.368 .

Estructura genética: De acuerdo con los valores de $\mathrm{F}_{\text {IS }}$ y $\mathrm{F}_{\text {IT }}(0.451$ y 0.504 respectivamente), los cuatro grupos analizados tienen $45.1 \%$ de déficit de heterocigotos y teniendo en cuenta la población total, el déficit es de $50.4 \%$. El AMOVA, basado en el estadístico 
TABLA 3

Parámetros de diversidad genética en las poblaciones de $B$. henni analizadas

TABLE 3

Genetic diversity parameters in $B$. henni populations

\begin{tabular}{lccccc}
\multicolumn{1}{c}{ Población } & $\mathrm{NP}_{\mathrm{A}}$ & $\mathrm{A}_{\mathrm{P}}$ & $\mathrm{H}_{\mathrm{E}}$ (D.E.) & $\mathrm{H}_{\mathrm{O}}$ (D.E.) & $\mathrm{F}_{\mathrm{IS}}$ \\
Río Campoalegre (RC) & 8 & 34 & $0.689(0.050)$ & $0.439(0.062)$ & 0.368 \\
Río Risaralda (RR) & 7 & 13 & $0.628(0.072)$ & $0.399(0.080)$ & 0.434 \\
Río Chinchiná (RCh) & 6 & 19 & $0.625(0.035)$ & $0.272(0.043)$ & 0.551 \\
Río Riofrío (RRf) & 5 & 10 & $0.515(0.055)$ & $0.241(0.030)$ & 0.479 \\
\hline
\end{tabular}

$\mathrm{R}_{\mathrm{ST}}$, detecto diferencias significativas $(\mathrm{P}<$ 0.05 ) y reveló que $90 \%$ de la variabilidad total encontrada se explicaba por variabilidad entre individuos dentro de poblaciones. Por su parte, la diferenciación entre poblaciones, basada en el estadístico $\mathrm{F}_{\mathrm{ST}}$, que expresa la reducción promedio en la heterocigosidad de una subpoblación generada por deriva genética, presentó un valor moderado y altamente significativo (0.09), por lo que solo el $9 \%$ de la varianza es atribuida a diferencias entre las subpoblaciones, por lo que se asume algún flujo genético entre ellas.

Los valores de $\mathrm{F}_{\mathrm{ST}}$ por parejas de poblaciones (Tabla 4), oscilaron entre 0.058 y 0.081 $\mathrm{y}$ todos fueron altamente significativos ( $\mathrm{P}$ $<0.001$ ), excepto entre RCh y RRf, con un valor bajo y no significativo, lo que podría deberse a algún programa de repoblación, aunque no hay documentación publicada al respecto. Finalmente, de acuerdo con la distancia genética de Nei (1972), la población más

TABLA 4

Distancias genéticas por parejas de poblaciones basadas en el parametro $\mathrm{F}_{\mathrm{ST}}$

TABLE 4

Genetic distances by population's pair base don $\mathrm{F}_{\mathrm{ST}}$ parameter

\begin{tabular}{lcccc} 
& RC & RR & RCh & RRf \\
RC & & & \\
RR & $0.058^{* *}$ & & \\
RCh & $0.074 * *$ & $0.066^{* *}$ & \\
RRf & $0.081^{* *}$ & $0.066^{* *}$ & $0.031 \mathrm{NS}$ \\
\hline
\end{tabular}

$* * \mathrm{P}<0.001 ; * \mathrm{P}<0.05$ divergente fue $\mathrm{RC}$, mientras que $\mathrm{RCh}$ y $\mathrm{RRf}$ fueron las más cercanas genéticamente entre ellas, como se muestra en la tabla 4. Aunque los valores de significancia del dendrograma fueron inferiores a $50 \%$, corresponden con los obtenidos con el estadístico $\mathrm{F}_{\mathrm{ST}}$, que fueron en su mayoría significativos.

En la figura 1 se representa gráficamente la estructura de la población (Pritchard et al., 2000) (Fig. 1.). Cada línea vertical representa un individuo que está dividido hasta en cuatro colores, según el número de k "Clusters". Los colores en cada individuo representan la proporción estimada de pertenencia a cada "Cluster". Se encontró la tendencia a cuatro poblaciones ancestrales. Según el coeficiente de pertenencia, las poblaciones son heterogéneas (Tabla 5). En RCh y RRf, cerca de $50 \%$ de los individuos provienen del mismo grupo (rojo), mientras que las poblaciones RR y RC tienen mayor heterogeneidad con respecto a posibles poblaciones ancestrales.

\section{DISCUSIÓN}

Las poblaciones de Brycon henni evaluadas en este estudio presentan una diferenciación genética moderada, posiblemente asociada al aislamiento físico actual entre ellas. Sin embargo, la menor distancia genética entre las poblaciones de RCh y RRf muestra cierta tendencia a pertenecer a un grupo común y la posible existencia de flujo génico entre ellas, posiblemente por influencia antrópica. Adicionalmente, el déficit de heterocigotos y el DL en muchos de los loci analizados sugieren 


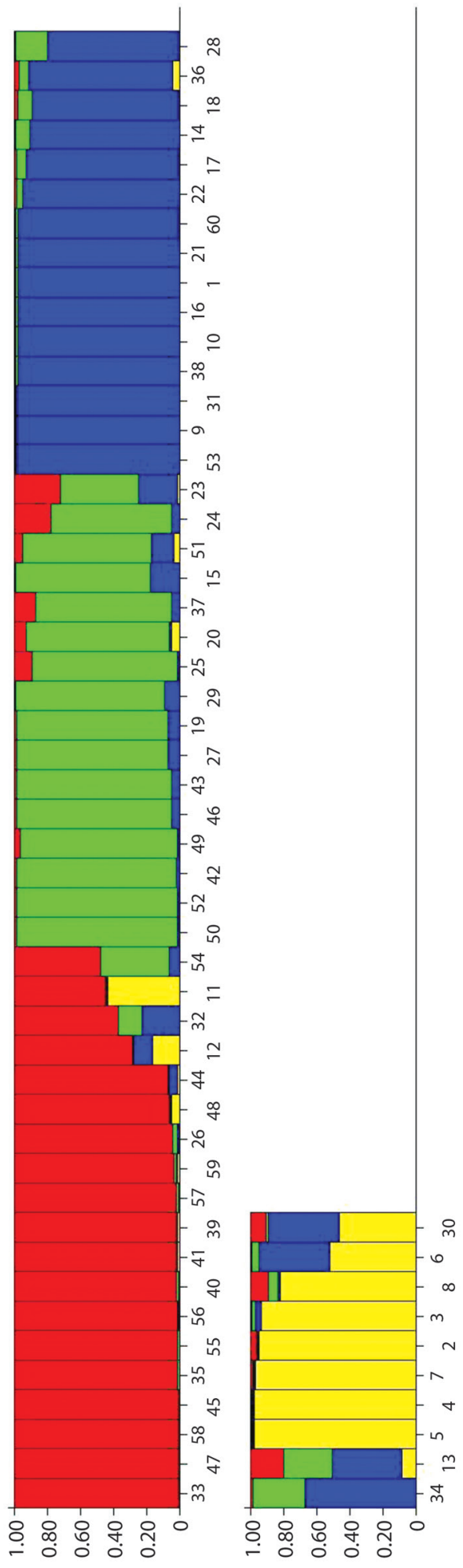

adicionalmente la influencia de endogamia, o muestreo de individuos emparentados, principalmente en las poblaciones de RCh y RRf.

Las amplias diferencias entre los valores de $\mathrm{N}_{\mathrm{A}}$ y $\mathrm{NE}_{\mathrm{A}}$ permiten inferir que muchos de los alelos encontrados para estas poblaciones son considerados como alelos raros, esto se confirma con el alto PIC encontrado en todos los marcadores, excepto en uno de ellos, dado que son interespecíficos y fueron seleccionados a partir de los más polimórficos empleados en estudios previos en el género Brycon. Por su parte, se presentó menor $\mathrm{H}_{\mathrm{O}}$ frente a otras especies de Brycon, como B. orthotaenia $(0.65$ - 0.73) según Sanches et al. (2012) y B. hilarii (0.59 - 0.61) según Sanches y Galetti (2012).

El alto $F_{\text {IT }}$ evidencia un marcado déficit de heterocigotos con respecto a la población total, una de las causas de ello puede ser la aparición de alelos nulos, confirmada por los valores moderados a altos de probabilidad de presencia en todos los loci, o a la reducción de los tamaños poblacionales poblaciones debido al deterioro ambiental. Según Eguiarte, Aguirre, Scheinvar, González, y Souza (2010), este coeficiente señala el efecto conjunto de la endogamia $\left(\mathrm{F}_{\mathrm{IS}}\right)$ y la deriva genética en la diferenciación de las poblaciones $\left(\mathrm{F}_{\mathrm{ST}}\right)$. Esta situación podría estar asociada al deterioro ambiental de las cuencas estudiadas, las cuales puede reducir las poblaciones de peces (Hurtado et al., 2011).

Aunque las poblaciones de $B$. henni estudiadas tienen algún nivel de diferenciación, el análisis sugiere que podría existir una tasa de

Fig. 1. Representación gráfica de estructura de la población. Cada línea vertical es un individuo y cada color representa un "cluster". La distribución de los colores representa la pertenencia de cada individuo a un grupo determinado. Individuos: 2-18 (RC), 19-36 (RR), 38-54 (RCh) y 55-72 (RRf).

Fig. 1. Graphic representation of population structure. Each vertical line is an individual and each color represents a "cluster". The distribution of the colors represents the belonging of each individual to a certain group. Individuals: 2-18 (RC), 19-36 (RR), 38-54 (RCh) and 55-72 (RRf). 
TABLA 5

Proporción de membresía de cada población en los cuatro "Clusters" identificados en el análisis bayesiano

TABLE 5

Membership proportion of each population in four clusters identified in Bayesian Analysis

\begin{tabular}{ccccc} 
Población & \multicolumn{4}{c}{ Cluster } \\
RC & 1 (azul) & 2 (amarillo) & 3 (rojo) & 4 (verde) \\
RR & 0.353 & 0.447 & 0.109 & 0.091 \\
RCh & 0.433 & 0.031 & 0.132 & 0.404 \\
RRf & 0.260 & 0.007 & 0.512 & 0.220 \\
\hline
\end{tabular}

migración considerable (2.86), que evidencia de un moderado flujo genético que sobrepasa los efectos de deriva e impide una mayor diferenciación local (Slatkin, 1994). Según Jiménez, Román-Valencia, \& Cardona (1998), la especie presenta movimientos migratorios entre el cauce principal y todos sus tributarios, los cuales pueden obedecer a la búsqueda de mejores condiciones del agua (temperatura, transparencia). Aunque estas migraciones no están necesariamente asociadas a la reproducción (Perdomo, 1978), por lo que la construcción de embalses y el deterioro de la calidad de agua de los corredores fluviales puede afectar sus migraciones. Las cuencas de los ríos Risaralda, Riofrío, y Chinchiná, son receptoras de aguas residuales de una gran cantidad de centros urbanos y el agua contiene una alta carga de contaminantes como metales pesados y residuos de insumos agrícolas (CVC, 2004) que podrían estar afectando las poblaciones naturales de $B$. henni.

Además de la presencia de alelos nulos, el exceso de homocigotos también está definido por efectos de endogamia al interior de las poblaciones, dado el alto $\mathrm{F}_{\mathrm{IS}}$, o, de una forma menos probable, por una mezcla de individuos de varias unidades panmícticas, con frecuencias alélicas diferentes (efecto Wahlund), que provoca un aumento de genotipos homocigotos, en las poblaciones analizadas (Pardo, Morales, \& Cavadia, 2014) y que en el presente estudio pudo producirse como un artefacto de muestreo por realizar trampeos en diferentes sitios de los ríos con la consecuente toma de animales de diferentes agrupaciones genéticas. Adicionalmente, con la técnica empleada, la presencia de alelos nulos se debe generalmente a mutaciones en las secuencias que flanquean la región microsatélite, las cuales sirven como sitio de unión a los partidores diseñados para producir la amplificación por PCR, por lo cual un genotipo heterocigoto puede ser registrado erróneamente como homocigoto (Pemberton, Slate, Bancroft \& Barrett, 1995).

Los valores de $\mathrm{F}_{\mathrm{IS}}$ encontrados en el presente estudio son superiores a los encontrados en otras especies de Brycon, como B. orbignyanus en Brasil, con -0.116 a -0.242 (Lopera et al., 2010) y -0.489 a -0.224 (Rodriguez et al., 2010), B. hilarii, con 0.096 a -0.109 (Sanches \& Galetti 2012) y B. orthotaenia, de 0.12 (Sanches et al., 2012), pero similares a los datos reportados por Barroso et al., (2005) para poblaciones de B. opalinus de Río Preto, Brasil, con 0.148 a 0.414 . En cuanto al $\mathrm{NP}_{\mathrm{A}}$ encontrado en este estudio, se obtuvieron datos semejantes al determinado por Sanches et al. (2012) en B. orthotaenia en el río San Francisco, en Brasil (5.6 a 7.2 alelos) y la $\mathrm{R}_{\mathrm{A}}$ encontrada presentó rango de valores más amplio que los hallados en especies como $B$. orthotaenia y B. hilarii, de 5.48 a 6.44 y 5.20 a 5.66 , respectivamente (Sanches et al., 2012, Sanches, \& Galetti, 2012).

La diversidad genética de muchas poblaciones de peces es muy variable, dependiendo 
de la especie, la ubicación del río y las presiones en cada ambiente (Povh et al., 2008). La mayor variabilidad genética se encontró en el $\mathrm{RC}$, cuenca con el mayor número de $\mathrm{A}_{\mathrm{P}} \mathrm{y}$ población más diferenciada; esta cuenca tiene aproximadamente $46.9 \%$ de su territorio bajo alguna categoría de conservación y las comunidades que rodean el río lo han protegido, evitando que personas foráneas realicen actividades pesqueras (Castaño \& Carranza, 2015).

Por otra parte, los valores más bajos de variabilidad genética en las otras poblaciones pueden ser consecuencia además, de la deposición de agua residual urbana e industrial y a la sobrepesca de la especie en dichos ríos, tal como lo sugirió Pineda et al., (2007) y Hurtado et al., (2011) en cuencas hidrográficas del departamento de Antioquia. Estos autores concluyeron que los efectos antrópicos de la sobrepesca, construcción de presas, deforestación y contaminación del agua, han contribuido al aislamiento de ciertos grupos, generando un alto nivel de estructura genética y un $50-70$ $\%$ de polimorfismo. Los resultados actuales mostraron que las poblaciones de B. henni evaluadas tienen un nivel moderado pero altamente significativo de estructura genética con valores de diversidad genética que lo convierten en un valioso recurso que debe ser preservado. Actualmente no hay informes oficiales de monitoreo del estado de las poblaciones de peces en estas cuencas; sin embargo, los resultados sugieren que las poblaciones pueden estar alcanzando valores críticos de baja densidad, lo cual pone en riesgo su conservación.

\section{AGRADECIMIENTOS}

Agradecemos a Vanessa Ruiz, Pablo Alejandro Hincapié y Oscar Eduardo Molano por su apoyo en el trabajo de campo. Heiber Cárdenas revisó el manuscrito preliminar e hizo valiosas sugerencias a la versión definitiva. Los autores agradecen las sugerencias de dos revisores anónimos. Este trabajo fue apoyado por el convenio entre la Autoridad Nacional de Acuacultura y Pesca de Colombia - AUNAP y la Corporación Universitaria Santa Rosa de Cabal - UNISARC, Convenio especial de cooperación de actividades científicas y tecnológicas No. 000226 de 11 de septiembre de 2015. Esta es una contribución del grupo de investigación Biología de la Conservación y Biotecnología de UNISARC.

\section{RESUMEN}

Las especies de peces en Colombia están sujetas a procesos de sobrepesca, introducción de especies exóticas, deterioro de hábitat y fragmentación de ecosistemas, con la consecuente pérdida de conectividad que limita el flujo genético y pudiera llevar a las poblaciones a la extinción. En el presente trabajo se analizó la estructura genética poblacional de Brycon henni, un pez de importancia económica y ecológica, en cuatro cuencas de la región Andina colombiana, mediante el uso de diez marcadores microsatélites en 60 muestras de los ríos Risaralda (RR), Campoalegre (RC), Riofrío (RRf) y Chinchiná ( $R C h)$. Se encontró un total de 136 alelos. Con excepción del marcador BoM12, todos fueron altamente informativos y polimórficos y en todos se presentó desviación significativa $(\mathrm{P}<0.05)$ del equilibrio Hardy Weinberg. En RC se observó el mayor número de alelos (81), Número promedio de alelos (8) y alelos privados (34), además una $\mathrm{H}_{\mathrm{E}}$ de $0.689 \pm 0.05$. En general, hubo un marcado déficit de heterocigotos con respecto a la población total $\left(\mathrm{F}_{\mathrm{IT}}=0.562\right)$, dentro de las subpoblaciones $\left(\mathrm{F}_{\mathrm{IS}}=0.526\right)$ y moderada, pero altamente significativa estructura genética $\left(\mathrm{F}_{\mathrm{ST}}=0.07\right)$. Según la distancia estándar de Nei, la población más divergente fue la del $\mathrm{RC}$ con un $\mathrm{k}=4$, se encontró tendencia a cuatro poblaciones ancestrales. Los resultados sugieren que el flujo genético entre poblaciones naturales de $B$. henni es limitado, lo cual puede tener efectos negativos sobre su conservación. Los resultados sugieren que las poblaciones pueden estar alcanzando valores críticos de baja densidad, lo cual pone en riesgo su conservación.

Palabras clave: alelo; antrópico; marcador codominante; región andina; sobrepesca.

\section{REFERENCIAS}

Aguirre, M., \& Muñoz, L. E. (2015). Evaluación de dos niveles de proteína en dietas para juveniles de sabaleta Brycon henni (Eigenmann, 1913). Revista Colombiana de Investigaciones Agroindustriales, 2, 61-68.

Balloux, F., \& Lugon-Moulin N. (2002). The estimation of population differentiation with microsatellite markers. Molecular Ecology, 11, 155-165.

Barroso, R., Hilsdorf, A., Moreira, H., Mello, A., Guimarães, S., Cabello, P., \& Traub, Y. (2003). 
Identification and characterization of microsatellites loci in Brycon opalinus (Cuvier, 1819) (Characiforme, Characidae, Bryconiae). Molecular Ecology Notes, 3, 297-298.

Barroso, R. Hilsdorf, A., Moreira, H., Cabellod, P., \& Traub, Y. (2005). Genetic diversity of wild and cultured populations of Brycon opalinus (Cuvier, 1819) (Characiforme, Characidae, Bryconiae) using microsatellites. Aquaculture, 247, 51-65.

Botero, A., \& Ramirez, H. (2011). Ecología trófica de la Sabaleta Brycon henni (Pisces: Characidae) en el río Portugal de Piedras, Alto Cauca, Colombia. Revista MVZ Córdoba, 16, 2349-2355.

Botstein, D., White, R., Skolnick, M., \& Davis, R. (1980). Construction of a genetic linkage map in man using restriction fragment length polymorphisms. American Journal of Human Genetics, 32, 314-331.

Castaño, J. H., \& Carranza, J. A. (2015). Campoalegre: Biodiversidad en un paisaje rural andino de Risaralda. Santa Rosa de Cabal, Colombia: UNISARC - CARDER.

Chacón, L., Angel, M., Serna, C., Echavarria, A., \& Velez, D. (2015). Maduración gonadal en hembras de sabaleta (Brycon henni) y su relación con variables medioambientales. Revista Politécnica, 1, 95-104.

CVC - (Corporación Autónoma Regional Del Valle Del Cauca). (2004). Caracterización de la calidad del agua del río Canca y sus tributarios mediante la utilización de índices de calidad y contaminación. Cali, Colombia: Universidad del Valle - Facultad de Ingeniería.

David, D., Vásquez, G., Ruiz, T., \& Olivera, M. (2008). Caracterización citogenética del pez neotropical Brycon henni (Pisces: Characidae). Revista de Biología Tropical, 56, 1619-1628.

De Miranda, L., Caldart, F., Muniz, C., Stephanie, P., Cardoso, A., \& Cavalcante, D. (2014). Estudio genético da piraputanga (Brycon microlepis). Biodiversidade, 13, 36-47.

Earl, D., \& Vonholdt, B. (2012). Structure harvester: a website and program for visualizing STRUCTURE output and implementing the Evanno method. Conservation Genetics Resources, 4, 359-361.

Ellegren, H. (2004). Microsatellites: simple sequences with complex evolution. Nature Reviews Genetics, 5(6), 435-445. DOI:10.1038/nrg1348

Eguiarte, L., Aguirre, E., Scheinvar, E., González, A., \& Souza, V. (2010). Flujo génico, diferenciación y estructura genética de las poblaciones, con ejemplos en especies de plantas mexicanas. México: Universidad Nacional Autónoma de México.
Evanno, G., Regnaut, S., \& Goudet, J. (2005). Detecting the number of clusters of individuals using the software STRUCTURE: a simulation study. Molecular Ecology, 14, 2611-2620.

Excoffier L., Laval, A., \& Scheneider, S. (2005). Arlequin ver 3.1: an integrated software package for population genetics data analysis. Evolutionary Bioinformatics, 1, 47-50.

Gastric, V., Bernatchez, L., Belkhir, K., \& Bonhome, F. (2002). Heterozygote deficiencies in small sacustrine populations of brook charr Salvelinus fontinalis Mitchill (Pices, Salmonidae): a test of alternative hypotheses. Heredity, 89, 27-35.

Goudet, J. (2002). FSTAT software. Recuperado de http:// www2.unil.ch/popgen/softwares/fstat.htm

Hurtado, J., Mancera, N., \& Saldamando, C. (2011). Variabilidad genética de Brycon henni (Characiformes: Characidae) en la cuenca media de los ríos Nare y Guatapé, sistema Río Magdalena, Colombia. Revista de Biología Tropical, 59, 269-282.

Jiménez, J., Román-Valencia, C., \& Cardona, M. (1998). Distribución y constancia de las comunidades de peces en la quebrada San Pablo, cuenca del Río La Paila, Alto Cauca, Colombia. Actualidades Biológicas, 20(68), 21-27.

Kumar, A. Y., Tomar, S., Kumar, A., \& Singh, J. 2017. Importance of Molecular Markers in Livestock Improvement: A Review. International Journal of Agriculture Innovations and Research, 5(4), 2319-1473.

Lemus, C., Ulloa, R., Ramos, F., \& Alonso, R. (2001). Genetic analysis of Mexican hairless pig populations. Journal of Animal Science, 79, 3021-3026.

Lenis, G., Restrepo, L., Rivera, J., Monsalve, F., \& Cruz, P. (2009). Reproducción inducida y producción de alevinos de Sabaleta Brycon henni: determinación del tiempo de latencia utilizando extracto de hipófisis de carpa. Revista Colombiana de Ciencias Pecuarias, 22, 143-155.

Lenis, G., Restrepo, L., \& Cruz, P. (2009). Evaluación de tres protocolos de tratamiento hormonal sobre el diámetro de ovocitos de sabaleta Brycon henni. Revista Colombiana de Ciencias Pecuarias, 22, 131-142.

Lenis, G., Cruz, P., \& David, C. (2015). Reproducción inducida de la sabaleta Brycon henni. Revisión bibliográfica. Revista Lasallista de Investigaciones, 12, 211-220.

Lopera, M., Vargas, L., Nardez, R., Pereira, R., Aparecido, J., Streit, P., \& Gomes, P. (2010). Diversidad genética y contribución reproductiva de una progenie de Brycon orbignyanus en el sistema reproductivo seminatural, usando marcadores microsatélites. Agrociencia, 44, 171-181. 
Maldonado, J., Ortega, A., Usma, J., Galvis, G., Villa, F., Vásquez, L., Prada, S., \& Ardila, C. (2005). Peces de los Andes de Colombia. Bogotá, Colombia: Instituto de Investigación de Recursos Biológicos Alexander von Humboldt.

Mancera, N., Marquez, E., \& Hurtado, J. (2013). Uso de citogenética y técnicas moleculares en estudios de diversidad genética en peces colombianos. En J. Zamora, A. Ortega, \& C. Ortiz (Eds.), Biología molecular aplicada a la producción animal y la conservación de especies silvestres (pp. 237-312). Colombia: Centro de publicaciones Universidad Nacional de Colombia Sede Medellín.

Matsumoto, C. \& Silva, A. (2009). Microsatellite variation and population genetic structure of a neotropical endangered Bryconinae species Brycon insignis (Steindachner, 1877): implications for its conservation and sustainable management. Neotropical Ichthyology, 7, 395-402.

Miller, M. (1997). Tools for Population Genetic Analyses (TFPGA), 1.3.A windows program for the analyses of allozyme and molecular population genetic data. Utah State University, Utah, USA. Recuperado de http://bioweb.usu.edu/mpmbio/tfpga.asp

Mojica, J. I., Usma, J. S., Álvarez-León, R., \& Lasso, C. A. (Eds.). (2012). Libro rojo de peces dulceacuícolas de Colombia. Bogotá, D. C., Colombia: Instituto de Investigación de Recursos Biológicos Alexander von Humboldt, Instituto de Ciencias Naturales de la Universidad Nacional de Colombia, WWF Colombia y Universidad de Manizales.

Montoya, A., Carrillo, L., \& Olivera, M. (2006a). Algunos aspectos biológicos y del manejo en cautiverio de la Sabaleta Brycon henni, Eigenmann, 1913 (Pisces: Characidae). Revista Colombiana de Ciencias Pecuarias, $19,180-186$

Montoya, A., Tabares, C., Echeverri, A., Arboleda, L., \& Olivera, M. (2006b). Descripción anatómica e histológica de las gónadas en Sabaleta (Brycon henni, Eigenmann 1913) Revista Colombiana de Ciencias Pecuarias, 19, 187-196.

Moreira, F., Polo, E., Silva, M., \& Menezes, G. (2015). Optimization of heterologous microsatellites in piracanjuba. Pesquisa Agropecuária Brasileira, 50, 1236-1239.

Muñoz, F., Villamarín, J., \& Londoño, L. (2016). Chromosomal differences in Sabaletas (Brycon henni) from the upper basins of the Cauca and Patia rivers. Revista CES Medicina Veterinaria y Zootecnia, 11, 62-71.

Nei, M. (1972). Genetic distance between populations. American Naturalist, 106, 283-292.

Palma, M., González, F., Romo, H., Ruiz, E., \& Fuentealba, C. (2007). Contamination effects on genetic diversity of Mazzaella laminarioides (bory) fredericq (Gigartinales, Rhodophyta) on bays. Gayana Botánica, 64(1), 24-32.

Pardo, P. E., Morales, J., \& Cavadia, M. T. (2014). Estudio de la diversidad genética de la población de gato doméstico (Felis catus) en Montería, Colombia. Bistua, 12(2), 35-47.

Park, S. (2001). The excel Microsatellite-Toolkit (versions3.1). Animal Genomics Laboratory, UCD, Ireland. Recuperado de http://animalgenomics.ucd.ie/ sdepark/ms-tookit

Peakall, R. \& Smouse, P. (2006). GENALEX 6: Genetic analysis in Excel. Population genetic software for teaching and research. Molecular Ecology Notes, 6, 288-295.

Perdomo, J. (1978). La Sabaleta (Brycon henni) observaciones bioecológicas y su importancia como especie de cultivo. Revista Divulgación Pesquera, 11(1), $1-46$.

Pemberton, J. M., Slate, J., Bancroft, D. R., \& Barrett, J. A. (1995). Nonamplifying alleles at microsatellite loci: a caution for parentage and population studies. Molecular Ecology, 4(2), 249-252.

Pineda, H., Arboleda, L., Echeverry, A., Urcuqui, S., Pareja, S., Olivera, M., \& Builes, J. (2007). Caracterización de la diversidad genética en el pez Brycon henni (Characiformes: Characidae) en Colombia central por medio de marcadores RAPD. Revista de Biología Tropical, 55, 1025-1035.

Piorski, N., Sanches, A., Carvalho, L., Hatanaka, T., Carri1lo, M., Freitas, P., \& Galetti, P. (2008). Contribution of conservation genetics in assessing neotropical freshwater fish biodiversity. Brazilian Journal of Biology, 68, 1039-1050.

Pritchard, J., Stephens, M., \& Donnelly, P. (2000). Inference of population structure using multilocus genotype data. Genetics, 155, 945-959.

Povh, J., Lopera, N., Ribeiro, R., Lupchinski, E., Gomes, P., \& Lopes, T. (2008). Monitoreo genético en programas de repoblamiento de peces mediante marcadores moleculares. Ciencia e Investigación Agraria, 35(1), 5-15.

Rodríguez, M., Lopera, N., Pereira, R., Aparecido, J., Vargas, L., Nardez, R., \& Bespalhok, C. (2010). Diversidad genética de piracanjuba usada en programas de repoblación con marcadores microsatélites. Pesquisa Agropecuária Brasileira, 45, 56-63.

Sanches, A., \& Galetti, P. Jr. (2006). Microsatellites loci isolated in the freshwater fish Bryconhilarii. Molecular Ecology Notes, 6, 1045-1046. 
Sanches, A., Galetti, P. Jr., Galzerani, F., Derazo, J., Cutilak, B., \& Hatanaka, T. (2012). Genetic population structure of two migratory freshwater fish species (Brycon orthotaenia and Prochilodus argenteus) from the São Francisco Riverin Brazil and its significance for conservation. Latin American Journal of Aquatic Research, 40, 177-186.

Sanches, A., \& Galetti, P. Jr. (2012). Population genetic structure revealed by a school of the freshwater migratory fish, Brycon hilarii. Latin American Journal of Aquatic Research, 40, 408-417.

Slatkin, M. (1994). Gene flow and population structure. In L. Real (Ed.). Ecological Genetics. Princeton, N.Y., USA: Princeton University Press.

Silva, A., Oliveira, C., Thadeo De Lima, F., \& Matsumoto, C. (2008). A phylogenetic analysis of Brycon and Henochilus (Characiformes, Characidae, Bryconinae) based on the mitochondrial gene 16S rRNA. Genetics and Molecular Biology, 31, 366-371.

Tabares, C., Montoya, A., Arboleda, L., Echeverri, A., Restrepo, L., \& Olivera, M. (2006). Efecto de la pluviosidad y el brillo solar sobre la producción y características del semen en el pez Brycon henni (Pisces: Characidae). Revista de Biología Tropical, 54, 179-187.

Tabares, J., Ruiz, T., Arboleda, L., \& Olivera, M. (2007). Effect of some ions on sperm activation in Bryconhenni (Eigenmann 1913). Acta Biológica Colombiana, 12, 87-98.

Taggart, J., Hynes, R., Prodohl, P., \& Fergusson, A. (1992). A simplified protocol for routine total DNA isolation from salmonid fishes. Journal of Fish Biology, 40, 963-965. 